\title{
Anisotropic regularity conditions for the suitable weak solutions to the 3d Navier-Stokes equations
}

\author{
Yanqing Wang* and Gang $\mathrm{Wu}^{\dagger}$
}

\begin{abstract}
We are concerned with the problem, originated from Seregin [18, 19, 20], what are minimal sufficiently conditions for the regularity of suitable weak solutions to the $3 \mathrm{~d}$ Naiver-Stokes equations. We prove some interior regularity criteria, in terms of either one component of the velocity with sufficiently small local scaled norm and the rest part with bounded local scaled norm, or horizontal part of the vorticity with sufficiently small local scaled norm and the vertical part with bounded local scaled norm. It is also shown that only the smallness on the local scaled $L^{2}$ norm of horizontal gradient without any other condition on the vertical gradient can still ensure the regularity of suitable weak solutions. All these conclusions improve pervious results on the local scaled norm type regularity conditions.
\end{abstract}

MSC(2000): 35Q30, 35A02.

Keywords: Navier-Stokes equations, suitable weak solutions, regularity.

\section{Introduction}

In this paper we consider the following classical incompressible 3d Navier-Stokes equations

$$
u_{t}-\Delta u+u \cdot \nabla u+\nabla \Pi=0, \operatorname{div} u=0,(x, t) \in \Omega \times(0, T),\left.u\right|_{\partial \Omega \times(0, T)}=0,
$$

where $\Omega \subseteq \mathbb{R}^{3}$ is a bounded regular domain, the vector field $u$ denotes velocity of the flow, the scalar function $\Pi$ stands for pressure of the fluid. The initial data $u(0)$ is also divergence-free.

In 1970s, Scheffer [14, 15, 16] introduced the concept of the suitable weak solutions, later developed by Caffarelli, Kohn, Nirenberg [2] and Lin [9], to the 3d Naiver-Stokes equations. In contrast to the usual Leray-Hopf weak solutions equipped with the energy inequality, the suitable weak solutions enjoy the following local energy inequality (inverse-Sobolev

${ }^{*}$ School of Mathematical Sciences, Capital Normal University, Beijing 100048, PR China. Email: wangyanqing20056@gmail.com.

${ }^{\dagger}$ School of Mathematical Sciences, University of Chinese Academy of Sciences, Beijing 100049, PR China. Email: wugangmaths@gmail.com. 
inequality)

$$
\begin{aligned}
& \int_{\Omega}\left|u\left(t^{\prime}, x\right)\right|^{2} \phi d x+2 \int_{t}^{t^{\prime}} \int_{\Omega}|\nabla u(s, x)|^{2} \phi d x d s \\
\leq & \int_{t}^{t^{\prime}} \int_{\Omega}|u(s, x)|^{2}\left(\partial_{t} \phi+\Delta \phi\right) d x d s+\int_{t}^{t^{\prime}} \int_{\Omega}\left(|u(s, x)|^{2}+2|\Pi(s, x)|\right) u(s, x) \cdot \nabla \phi d x d s,
\end{aligned}
$$

for any non-negative function $\phi \in C_{0}^{\infty}\left(\Omega \times\left(t, t^{\prime}\right)\right)$.

We now recall the definition of suitable weak solutions for the Navier-Stokes equations.

Definition (Suitable weak solutions). A pair $(u, \Pi)$ is said to be a suitable weak solution to the Navier-Stokes equations (1.1), provided the following conditions are satisfied

(i) $u \in L^{\infty}\left(t, t^{\prime} ; L^{2}(\Omega)\right) \cap L^{2}\left(t, t^{\prime} ; W^{1,2}(\Omega)\right), \quad \Pi \in L^{5 / 3}\left(t, t^{\prime} ; L^{5 / 3}(\Omega)\right)$.

(ii) $(u, \Pi)$ solves $(1.1)$ in $\Omega \times\left(t, t^{\prime}\right)$ in the sense of distributions.

(iii) $(u, \Pi)$ satisfies the local energy inequality (1.2) .

For the sake of statements, we denote by $\omega=\operatorname{curl} u$ the vorticity of the flow, and by $u_{h}=\left(u_{1}, u_{2}, 0\right)$ and $\omega_{h}=\left(\omega_{1}, \omega_{2}, 0\right)$ the horizontal part of the velocity and the vorticity, respectively. Similarly, the horizontal gradient operator and vertical gradient operator are denoted by $\nabla_{h}=\left(\partial_{1}, \partial_{2}, 0\right)$ and $\nabla_{3}=\left(0,0, \partial_{3}\right)$, respectively. Throughout this paper, we also set

$$
\begin{array}{ll}
B(x, r)=\left\{y \in \mathbb{R}^{3}|| x-y \mid \leq r\right\}, & B(r)=B(0, r), \\
Q(x, t, r)=B(x, r) \times\left(t-r^{2}, t\right), & Q(r)=Q(0,0, r) .
\end{array}
$$

A point is said to be a regular point to (1.1) if $u$ is bounded in some neighborhood of this point. The rest points will be called singular points. Making full use of the local energy inequality (1.2), Scheffer [14, 15, 16] could estimate the size of the potential spacetime singular points set of suitable weak solutions. The optimal estimate of the Hausdorff dimension of the possible singular points set was obtained by Caffarelli, Kohn, Nirenberg [2] via establishing the following regularity criterion: there is an absolute constant $\varepsilon_{1}$ such that, if

$$
\limsup _{r \rightarrow 0} \frac{1}{r} \iint_{Q(r)}|\nabla u|^{2} d x d t \leq \varepsilon_{1}
$$

then $(0,0)$ is a regular point.

Since then, there has been much effort to study the partial regularity of suitable weak solutions, see, for example, 4, 6, 7, 8, 9, 12, 17, 18, 19, 20, 23, 24, 27, 28]. On one hand, various kinds of alternative approach to the partial regularity theory of 3d Navier-stokes equations are developed. By means of blow-up procedure and compact method, Lin [9] gave a simple proof of Caffarelli-Kohn-Nirenberg's theorem. See also Ladyzenskaja and Seregin [8] for more details. Applying the De Giorgi iteration argument to the NavierStokes equations, Vasseur [24] rebuilt the main results in [2]. It should be pointed out that the final task of all these proof is to prove that (1.3) holds. Very recently, an analogue of Caffarelli-Kohn-Nirenberg's theorem in [2] for the suitable weak solutions to the 4d NavierStokes equations is proved in [28], where an analogous regularity condition (1.3) is verified, see also Dong and Gu [6]. However, it is not known whether the Caffarelli-Kohn-Nirenberg 
type regularity criterion (1.3) is valid for the higher dimensional Naiver-Stokes equations. On the other hand, sufficient regularity conditions on local scaled norm similar to (1.3) are generalized and improved, see, for example, [7, 17, 18, 19, 20, 23, 27]. Particularly, Tian and Xin [23] established the following criteria: there exists an absolute constant $\varepsilon_{2}$ such that, if the suitable weak solution $u$ satisfies

$$
\limsup _{r \rightarrow 0} \frac{1}{r} \iint_{Q(r)}|\omega|^{2} d x d t \leq \varepsilon_{2} \quad \text { or } \quad \limsup _{r \rightarrow 0} \frac{1}{r^{2}} \iint_{Q(r)}|u|^{3} d x d t \leq \varepsilon_{2},
$$

then $(0,0)$ is a regular point. Later, the regularity conditions (1.3) and (1.4) are further generalized and strengthened by Gustafson, Kang and Tsai in [7] to

$$
\limsup _{r \rightarrow 0} r^{1-\frac{3}{p}-\frac{2}{q}}\left(\int_{-r^{2}}^{0}\left(\int_{B(r)}|u|^{p} d x\right)^{\frac{q}{p}} d s\right)^{\frac{1}{q}} \leq \varepsilon_{3}, \quad 1 \leq \frac{3}{p}+\frac{2}{q} \leq 2,1 \leq p, q \leq \infty ;
$$

or

$$
\limsup _{r \rightarrow 0} r^{2-\frac{3}{p}-\frac{2}{q}}\left(\int_{-r^{2}}^{0}\left(\int_{B(r)}|\nabla u|^{p} d x\right)^{\frac{q}{p}} d s\right)^{\frac{1}{q}} \leq \varepsilon_{3}, \quad 2 \leq \frac{3}{p}+\frac{2}{q} \leq 3,1 \leq p, q \leq \infty
$$

or

$$
\begin{gathered}
\limsup _{r \rightarrow 0} r^{2-\frac{3}{p}-\frac{2}{q}}\left(\int_{-r^{2}}^{0}\left(\int_{B(r)}|\omega|^{p} d x\right)^{\frac{q}{p}} d s\right)^{\frac{1}{q}} \leq \varepsilon_{3}, \\
2 \leq \frac{3}{p}+\frac{2}{q} \leq 3,1 \leq p, q \leq \infty,(p, q) \neq(1, \infty)
\end{gathered}
$$

where $\varepsilon_{3}$ is an absolute constant. For other versions of the local scaled norm type regularity conditions, we refer the reader to [17, 18] and references therein.

In [18, 19, 20], Seregin began to address the problem what are minimal conditions which guarantee the regularity of suitable weak solutions. To be more precisely, some results in [20] read as follows. For any $M>0$, there exists a positive number $\varepsilon_{4}(M)$ such that, if

$$
\limsup _{r \rightarrow 0} \frac{1}{r} \iint_{Q(r)}|\nabla u|^{2} d x d t \leq M \text { and } \liminf _{r \rightarrow 0} \frac{1}{r} \iint_{Q(r)}\left|\nabla_{3} u\right|^{2} d x d t \leq \varepsilon_{4}(M),
$$

or

$$
\limsup _{r \rightarrow 0} \frac{1}{r} \iint_{Q(r)}|u|^{3} d x d t \leq M \text { and } \liminf _{r \rightarrow 0} \frac{1}{r} \iint_{Q(r)}|u|^{3} d x d t \leq \varepsilon_{4}(M),
$$

then $(0,0)$ is a regular point. This improved the famous Caffarelli-Kohn-Nirenbergs condition. The proof is mainly based on used a blow-up procedure. Utilizing this method, Wang and Zhang [27] recently showed that the smallness of horizontal part of the velocity is enough to ensure the regularity of suitable weak solutions, namely, for any constant $M>0$, there is a positive number $\varepsilon_{5}(M)$ such that $u$ is regular at $(0,0)$ if one of the following conditions holds,

$$
\begin{array}{r}
\limsup _{r \rightarrow 0} r^{1-\frac{3}{p}-\frac{2}{q}}\left(\int_{-r^{2}}^{0}\left(\int_{B(r)}|u|^{p} d x\right)^{\frac{q}{p}} d s\right)^{\frac{1}{q}} \leq M \\
\text { and } \liminf _{r \rightarrow 0} r^{1-\frac{3}{p}-\frac{2}{q}}\left(\int_{-r^{2}}^{0}\left(\int_{B(r)}\left|u_{h}\right|^{p} d x\right)^{\frac{q}{p}} d s\right)^{\frac{1}{q}} \leq \varepsilon_{5}(M),
\end{array}
$$


with $1 \leq \frac{3}{p}+\frac{2}{q}<2,1<p, q \leq \infty$;

$$
\begin{array}{r}
\limsup _{r \rightarrow 0} r^{2-\frac{3}{p}-\frac{2}{q}}\left(\int_{-r^{2}}^{0}\left(\int_{B(r)}|\nabla u|^{p} d x\right)^{\frac{q}{p}} d s\right)^{\frac{1}{q}} \leq M \\
\text { and } \liminf _{r \rightarrow 0} r^{-\left(\frac{3}{p}+\frac{2}{q}-2\right)}\left(\int_{-r^{2}}^{0}\left(\int_{B(r)}\left|\nabla u_{h}\right|^{p} d x\right)^{\frac{q}{p}} d s\right)^{\frac{1}{q}} \leq \varepsilon_{5}(M),
\end{array}
$$

with $2 \leq \frac{3}{p}+\frac{2}{q}<3,1<p, q \leq \infty$.

The main objective of this paper is to refine the above regularity criteria for the regularity of the suitable weak solutions. Notice that the results obtained by Seregin [20] and Wang and Zhang [27] do not include the regularity criterion in terms of the vorticity which is one of most important physical quantities in fluid flows (see, for example, [1, 10]). Our first result is to establish a regular condition involving the smallness on the horizontal part of the vorticity. Secondly, we show that the smallness of horizontal part of the velocity in (1.8) and (1.9) can be reduced to the smallness of only one component of the velocity. It is worth noting that all previous sufficient conditions in [7, 20, 23, 27] involve full components of the velocity, vorticity or gradient of the velocity rather than genuine partial components. Our third result is to show that the smallness of horizontal gradient without any condition on the vertical gradient can still imply the regularity of suitable weak solutions.

Theorem 1.1. Suppose that the pair $(u, \Pi)$ be a suitable weak solution to (1.1) in $Q(1)$. For any constant $M>0$, there exists a positive constant $\varepsilon_{11}(M)$ such that if $\omega \in L^{p} L^{q}(Q(1))$,

$$
\limsup _{r \rightarrow 0} r^{2-\frac{3}{p}-\frac{2}{q}}\left(\int_{-r^{2}}^{0}\left(\int_{B(r)}|\omega|^{p} d x\right)^{\frac{q}{p}} d s\right)^{\frac{1}{q}} \leq M,
$$

and

$$
\liminf _{r \rightarrow 0} r^{2-\frac{3}{p}-\frac{2}{q}}\left(\int_{-r^{2}}^{0}\left(\int_{B(r)}\left|\omega_{h}\right|^{p} d x\right)^{\frac{q}{p}} d s\right)^{\frac{1}{q}} \leq \varepsilon_{11}(M),
$$

with $2 \leq 3 / p+2 / q<3,1<p, q \leq \infty$, then $(0,0)$ is a regular point.

This result is an extension of criterion (1.7). The proof relies on the blow-up method used in [20, 27]. The key point is how to gain the interior regularity for the suitable weak solution to the following system

$$
\left\{\begin{array}{l}
u_{t}-\Delta u+u \cdot \nabla u+\nabla \Pi=0 \\
\operatorname{div} u=0, \quad \omega_{h}=0
\end{array}\right.
$$

As a matter of fact, if two components of a vorticity belongs to $L_{x}^{p} L_{t}^{q}$ for $3 / p+2 / q \leq 2$, then the suitable weak solution of the Navier-Stokes equations is regular, which is shown by Chae, Kang and Lee in [4]. To make our paper more self-contained and more readable, we shall outline an alternative path to the proof of interior regularity for the system (1.12) in Appendix. We use elementary tools involving the special structure of equations (1.12) and the bootstrapping argument utilized by Serrin in [21]. We would like to note that we work on the velocity equations rather than the vorticity equations used in [21]. This together with the Biot-Savart law and the Calderón-Zygmund Theorem allows us to prove Theorem 1.1 . 
Theorem 1.2. Let $(u, \Pi)$ be a suitable weak solutions to (1.1) in $Q(1)$. For any constant $M>0$, there exists a positive constant $\varepsilon_{21}(M)\left(\right.$ or $\left.\varepsilon_{22}(M)\right)$ such that $(0,0)$ is regular point if

(1) $u \in L^{p} L^{q}(Q(1))$, with $1 \leq 3 / p+2 / q<2,1<p, q \leq \infty$,

$$
\limsup _{r \rightarrow 0} r^{1-\frac{3}{p}-\frac{2}{q}}\left(\int_{-r^{2}}^{0}\left(\int_{B(r)}|u|^{p} d x\right)^{\frac{q}{p}} d s\right)^{\frac{1}{q}} \leq M,
$$

and

$$
\liminf _{r \rightarrow 0} r^{1-\frac{3}{p}-\frac{2}{q}}\left(\int_{-r^{2}}^{0}\left(\int_{B(r)}\left|u_{3}\right|^{p} d x\right)^{\frac{q}{p}} d s\right)^{\frac{1}{q}} \leq \varepsilon_{21}(M) ;
$$

(2) $\nabla u \in L^{p} L^{q}(Q(1))$, with $2 \leq 3 / p+2 / q<3,1<p, q \leq \infty$,

$$
\limsup _{r \rightarrow 0} r^{2-\frac{3}{p}-\frac{2}{q}}\left(\int_{-r^{2}}^{0}\left(\int_{B(r)}|\nabla u|^{p} d x\right)^{\frac{q}{p}} d s\right)^{\frac{1}{q}} \leq M,
$$

and

$$
\liminf _{r \rightarrow 0} r^{2-\frac{3}{p}-\frac{2}{q}}\left(\int_{-r^{2}}^{0}\left(\int_{B(r)}\left|\nabla u_{3}\right|^{p} d x\right)^{\frac{q}{p}} d s\right)^{\frac{1}{q}} \leq \varepsilon_{22}(M)
$$

Notice that any suitable weak solution to the following system

$$
\left\{\begin{array}{l}
u_{t}-\Delta u+u \cdot \nabla u+\nabla \Pi=0, \\
\operatorname{div} u=0, \quad u_{3}=0
\end{array}\right.
$$

is bounded, which was proved by Neustupa and Penel in [12]. The result of [12] says that if any one component of the velocity, which is a suitable weak solutions to the $3 \mathrm{~d}$ NavierStokes equations, is bounded, then the velocity has no singular point. Then the proof will be done in the same spirit as Theorem 1.1.

With the first regular criterion in Theorem 1.2 in hand, following the path of Section 4 in [27], one can further extend the Ladyzhenskaya-Prodi-Serrin's criterion obtained there. We leave this to the interesting reader.

Now we turn to the third result in this paper. Note that the following version of Sobolev and Ladyzhenskaya's inequalities

$$
\|f\|_{L^{p}\left(\mathbb{R}^{3}\right)} \leq C\|f\|_{L^{2}\left(\mathbb{R}^{3}\right)}^{\frac{6-p}{2 p}}\left\|\partial_{1} f\right\|_{L^{2}\left(\mathbb{R}^{3}\right)}^{\frac{p-2}{2 p}}\left\|\partial_{2} f\right\|_{L^{2}\left(\mathbb{R}^{3}\right)}^{\frac{p-2}{2 p}}\left\|\partial_{3} f\right\|_{L^{2}\left(\mathbb{R}^{3}\right)}^{\frac{p-2}{2 p}}, \text { with } 2 \leq p \leq 6,
$$

are frequently used in the investigation of models of incompressible fluid mechanics. For instance, see [3, 5, 11, 29] and references therein. Here, we are able to prove the local version of these inequalities (see Lemma 2.5 for details), which entails the following theorem.

Theorem 1.3. There exists an absolute constant $\varepsilon_{31}$ with the following property. If $(u, \Pi)$ is a suitable weak solution and

$$
\limsup _{r \rightarrow 0} \frac{1}{r} \iint_{Q(r)}\left|\nabla_{h} u\right|^{2} d x d s \leq \varepsilon_{31}
$$

then $(0,0)$ is a regular point. 
This is an improvement of condition (1.3) given by Caffarelli, Kohn and Nirenberg in [2]. As a by-product of Theorem 1.3. Hölder's inequality and absolute continuity of Lebesgue's integral immediately yield that

Corollary 1.4. Suppose that $(u, \Pi)$ is a suitable weak solution to (1.1). If there exist a constant $r$ such that

$$
\nabla_{h} u \in L_{x}^{p} L_{t}^{q}(Q(r)), \text { with } \frac{3}{p}+\frac{2}{q} \leq 2,2 \leq p, q \leq \infty,
$$

then $(0,0)$ is regular point.

This paper is organized as follows. In the second section, we introduce some notations, recall some known conclusions and prove some auxiliary lemmas which will play a key role in the proof of our results. Section 3 contains the proofs of Theorem 1.1 and 1.2. Section 4 is devoted to prove Theorem 1.3. Finally, an appendix is dedicated to the proof of the regularity of the solution to system (1.12).

\section{Notations and some auxiliary lemmas}

For $p \in[1, \infty]$, the notation $L^{p}((0, T) ; X)$ stands for the set of measurable functions on the interval $(0, T)$ with values in $X$ and $\|f(t, \cdot)\|_{X}$ belongs to $L^{p}(0, T)$. For simplicity, we write $\|f\|_{L^{p} L^{q}(Q(r))}:=\|f\|_{L^{q}\left(-r^{2}, 0 ; L^{p}(B(r))\right)}$ and $\|f\|_{L^{p}(Q(r))}:=\|f\|_{L^{p} L^{p}(Q(r))}$. We will also use the summation convention on repeated indices. $C$ is an absolute constant which may be different from line to line unless otherwise stated.

Due to the scaling property of Navier-Stokes equations [2], we introduce the following dimensionless quantities

$$
\begin{array}{cc}
E_{p}(u, r)=\frac{1}{r^{5-p}} \iint_{Q(r)}|u|^{p} d x d t, & E_{*}(u, r)=\frac{1}{r} \iint_{Q(r)}|\nabla u|^{2} d x d t, \\
E(u, r)=\sup _{-r^{2} \leq t<0} \frac{1}{r} \int_{B(r)}|u|^{2} d x, & P_{q}(\Pi, r)=\frac{1}{r^{5-2 q}} \iint_{Q(r)}|\Pi|^{q} d x d t, \\
E_{*, h}(u, r)=\frac{1}{r} \iint_{Q(r)}\left|\nabla_{h} u\right|^{2} d x d t, & E_{*, 3}(u, r)=\frac{1}{r} \iint_{Q(r)}\left|\nabla_{3} u\right|^{2} d x d t, \\
W(\omega, r)=\frac{1}{r} \iint_{Q(r)}|\omega|^{2} d x d t, & W_{h}(\omega, r)=\frac{1}{r} \iint_{Q(r)}\left|\omega_{h}\right|^{2} d x d t, \\
E_{p, q}(u, r)=r^{1-\frac{3}{p}-\frac{2}{q}}\left(\int_{-r^{2}}^{0}\left(\int_{B(r)}|u|^{p} d x\right)^{\frac{q}{p}} d t\right)^{\frac{1}{q}}, \\
E_{* ; p, q}(u, r)=r^{2-\frac{3}{p}-\frac{2}{q}}\left(\int_{-r^{2}}^{0}\left(\int_{B(r)}|\nabla u|^{p} d x\right)^{\frac{q}{p}} d t\right)^{\frac{1}{q}}, \\
W_{p, q}(\omega, r)=r^{2-\frac{3}{p}-\frac{2}{q}}\left(\int_{-r^{2}}^{0}\left(\int_{B(r)}|\omega|^{p} d x\right)^{\frac{q}{p}} d t\right)^{\frac{1}{q}} .
\end{array}
$$

First, we recall some known results. 
Lemma 2.1. [19, 27] Let $(u, \Pi)$ be a suitable weak solution to (1.1) in $Q(1)$. Assume that, for any constant $M>0$ and $r \in(0,1]$, there hold

$$
r^{1-\frac{3}{p}-\frac{2}{q}}\left(\int_{-r^{2}}^{0}\left(\int_{B(r)}|u|^{p} d x\right)^{\frac{q}{p}} d s\right)^{\frac{1}{q}} \leq M \quad \text { with } \quad 1 \leq \frac{3}{p}+\frac{2}{q}<2,1<q \leq \infty
$$

or

$$
r^{2-\frac{3}{p}-\frac{2}{q}}\left(\int_{-r^{2}}^{0}\left(\int_{B(r)}|\nabla u|^{p} d x\right)^{\frac{q}{p}} d s\right)^{\frac{1}{q}} \leq M \quad \text { with } \quad 2 \leq \frac{3}{p}+\frac{2}{q}<3,1<p \leq \infty .
$$

Then, for any $r \in\left(0, \frac{1}{2}\right]$,

$$
E(u, r)+E_{*}(u, r)+P_{3 / 2}(\Pi, r) \leq C(p, q, M)\left(r^{1 / 2}\left(E_{3}(u, 1)+P_{3 / 2}(\Pi, 1)\right)+1\right) .
$$

Theorem 2.2. [8, 9] Let $(u, \Pi)$ be a suitable weak solution to (1.1) in $Q(1)$. There exists $\varepsilon_{0}>0$ such that if

$$
\iint_{Q(1)}|u|^{3}+|\Pi|^{3 / 2} d x d t \leq \varepsilon_{0}
$$

then $u$ is regular in $Q(1 / 2)$.

Secondly, we present a decay type estimate of the pressure. For the other versions of decay estimate for the pressure, we refer the reader to $[7,8,49,19,23,28$.

Lemma 2.3. For $0<\mu \leq \frac{1}{2} \rho$ and $1<q<\infty$, there is an absolute constant $C$ independent of $\mu$ and $\rho$ such that

$$
P_{q}(\Pi, \mu) \leq C\left(\frac{\rho}{\mu}\right)^{5-2 q} E_{2 q}(u, \rho)+C\left(\frac{\mu}{\rho}\right)^{2 q-2} P_{q}(\Pi, \rho) .
$$

Proof. We consider the usual cut-off function $\phi \in C_{0}^{\infty}(B(\rho))$ such that $\phi \equiv 1$ on $B\left(\frac{3}{4} \rho\right)$ with $0 \leq \phi \leq 1$ and $|\nabla \phi| \leq C \rho^{-1},\left|\nabla^{2} \phi\right| \leq C \rho^{-2}$.

Due to the pressure equation $\partial_{i} \partial_{i} \Pi=\partial_{i} \partial_{j}\left(u_{j} u_{i}\right)$, we may write

$$
\partial_{i} \partial_{i}(\Pi \phi)=-\phi \partial_{i} \partial_{j}\left(u_{j} u_{i}\right)+2 \partial_{i} \phi \partial_{i} \Pi+\Pi \partial_{i} \partial_{i} \phi
$$

from which it follows that for $x \in B\left(\frac{3}{4} \rho\right)$,

$$
\begin{aligned}
\Pi(x)= & \Gamma *\left\{-\phi \partial_{i} \partial_{j}\left(u_{j} u_{i}\right)+2 \partial_{i} \phi \partial_{i} \Pi+\Pi \partial_{i} \partial_{i} \phi\right\} \\
= & -\partial_{i} \partial_{j} \Gamma *\left(\phi u_{j} u_{i}\right) \\
& +2 \partial_{i} \Gamma *\left(\partial_{j} \phi u_{j} u_{i}\right)-\Gamma *\left(\partial_{i} \partial_{j} \phi u_{j} u_{i}\right) \\
& -2 \partial_{i} \Gamma *\left(\partial_{i} \phi \Pi\right)-\Gamma *\left(\partial_{i} \partial_{i} \phi \Pi\right) \\
= & : P_{1}(x)+P_{2}(x)+P_{3}(x),
\end{aligned}
$$

where $\Gamma$ stands for the normalized fundamental solution of Laplace's equation in $\mathbb{R}^{3}$.

Since $\phi(x)=1$ when $x \in B(\mu)$, we deduce that

$$
\Delta\left(P_{2}(x)+P_{3}(x)\right)=0, x \in B(\mu) .
$$


By the mean value property of harmonic functions, we have

$$
\begin{aligned}
\int_{B(\mu)}\left|P_{2}(x)+P_{3}(x)\right|^{q} d x & \leq C\left(\frac{\mu}{\rho}\right)^{3} \int_{B(\rho / 2)}\left|P_{2}(x)+P_{3}(x)\right|^{q} d x \\
& \leq C\left(\frac{\mu}{\rho}\right)^{3} \int_{B(\rho / 2)}\left|\Pi(x)-P_{1}(x)\right|^{q} d x \\
& \leq C\left(\frac{\mu}{\rho}\right)^{3} \int_{B(\rho / 2)}|\Pi(x)|^{q} d x+C \int_{B(\rho / 2)}\left|P_{1}(x)\right|^{q} d x
\end{aligned}
$$

where $C$ is independent of $\mu$ and $\rho$.

According to the classical Calderón-Zygmund Theorem, it is easy to get

$$
\int_{B(\mu)}\left|P_{1}(x)\right|^{q} d x \leq \int_{B(\rho / 2)}\left|P_{1}(x)\right|^{q} d x \leq C \int_{B(\rho)}|u|^{2 q} d x
$$

Collecting (2.6)-(2.8) yields

$$
\begin{aligned}
\int_{B(\mu)}|\Pi|^{q} d x & \leq C \int_{B(\mu)}\left|P_{1}\right|^{q}+\left(\left|P_{2}+P_{3}\right|\right)^{q} d x \\
& \leq C\left(\int_{B(\rho)}|u|^{2 q} d x\right)+C\left(\frac{\mu}{\rho}\right)^{3} \int_{B(\rho)}|\Pi|^{q} d x .
\end{aligned}
$$

Integrating this inequality in time gives

$$
\iint_{Q(\mu)}|\Pi|^{q} d x d s \leq C\left(\iint_{Q(\rho)}|u|^{2 q} d x d s\right)+C\left(\frac{\mu}{\rho}\right)^{3} \iint_{Q(\rho)}|\Pi|^{q} d x d s,
$$

which in turn implies the desired estimate (2.5).

The following lemma can be regarded as the localized version of the Biot-Savart law.

Lemma 2.4. Let $(u, \Pi)$ be a suitable weak solution to (1.1) in $Q(1)$. If $\omega \in L^{p} L^{q}(Q(1))$ and

$$
\limsup _{r \rightarrow 0} r^{2-\frac{3}{p}-\frac{2}{q}}\left(\int_{-r^{2}}^{0}\left(\int_{B(r)}|\omega|^{p} d x\right)^{\frac{q}{p}} d s\right)^{\frac{1}{q}} \leq M,
$$

with $2 \leq 3 / p+3 / q<3,1<p, q$, then there exist $r^{*} \leq 1 / 2$ and $p^{\prime}, q^{\prime}$ satisfying $2 \leq$ $3 / p^{\prime}+2 / q^{\prime}<3$ such that

$$
E_{*, p^{\prime}, q^{\prime}}(u, r) \leq C(M)
$$

for $0<r \leq r^{*}$.

Proof. By Hölder's inequality, for any $2 \leq \frac{3}{p}+\frac{2}{q}<3$, we can choose $p^{\prime} \leq p, q^{\prime} \leq q$ and $p^{\prime}<3$ such that $2 \leq \frac{3}{p^{\prime}}+\frac{2}{q^{\prime}}<3$ and

$$
W_{p^{\prime}, q^{\prime}}(\omega, r) \leq C W_{p, q}(\omega, r)
$$

Using usual cut-off function in equations $\Delta u=-\operatorname{curl} \omega$ together with the fact that $\operatorname{div} u=0$, and the Calderón-Zygmund Theorem, we can deduce that

$$
E_{*, p^{\prime}, q^{\prime}}(u, \mu) \leq C\left(\frac{\rho}{\mu}\right) W_{p^{\prime}, q^{\prime}}(\omega, \rho)+C\left(\frac{\mu}{\rho}\right)^{\frac{3}{p^{\prime}}-1} E_{*, p^{\prime}, q^{\prime}}(u, \rho),
$$


where $0<\mu<\frac{1}{2} \rho$. This process is similar to the derivation of (2.5) in Lemma 2.3, For details, see Lemma 3.6 in [7] or Lemma 3.6 in [23].

Applying the classical elliptic estimate to $\Delta u=-\operatorname{curl} \omega$, we infer that $\nabla u \in$ $L^{p^{\prime}} L^{q^{\prime}}(Q(1 / 2))$ and

$$
\|\nabla u\|_{L^{p^{\prime} L^{q^{\prime}}(Q(1 / 2))}} \leq C\|\omega\|_{L^{p^{\prime} L^{q^{\prime}}(Q(1))}}+C\|u\|_{L^{2} L^{\infty}(Q(1))} .
$$

With the help of this fact and the hypothesis (2.10), the desired estimate (2.11) can be derived by iterating (2.12). Similar iteration technique will be used in the proof of Theorem 1.3 in Section 4, see also [2, 23, 28].

The last lemma is concerned with a inequality which is a local version of Sobolev and Ladyzhenskaya's inequalities (1.17) and will play an important role in the proof of Theorem 1.3 .

Lemma 2.5. For $0<\sqrt{3} \mu \leq \rho$, there exists an absolute constant $C$ independent of $\mu$ and $\rho$ such that

$$
\begin{aligned}
E_{10 / 3}(u, \mu) \leq & C\left(\frac{\rho}{\mu}\right)^{5 / 3} E^{2 / 3}(u, \rho) E_{*, 3}^{1 / 3}(u, \rho) E_{*, h}^{2 / 3}(u, \rho) \\
& +C\left(\frac{\rho}{\mu}\right)^{5 / 3} E(u, \rho) E_{*, h}^{2 / 3}(u, \rho)+C\left(\frac{\mu}{\rho}\right)^{1 / 3} E_{10 / 3}(u, \rho) .
\end{aligned}
$$

Proof. Set

$$
\overline{u_{\sqrt{2} r}^{h}}=\frac{1}{2 \pi r^{2}} \iint_{x_{1}^{2}+x_{2}^{2}<2 r^{2}} u d x_{1} d x_{2}
$$

where $r \geq \mu$.

It is obvious that

$$
\int_{B(\mu)}|u|^{\frac{10}{3}} d x \leq C \int_{B(\mu)}\left|u-\overline{u_{\sqrt{2} r}^{h}}\right|^{\frac{10}{3}} d x+C \int_{B(\mu)} \mid{\overline{u_{\sqrt{2} r}^{h}}}^{\frac{10}{3}} d x
$$

On one hand, utilizing Hölder's inequality, we find that

$$
\begin{aligned}
\left.\int_{B(\mu)} \overline{u_{\sqrt{2} r}^{h}}\right|^{\frac{10}{3}} d x & \leq C \int_{\left|x_{3}\right|<\mu} d x_{3} \iint_{\left|x_{1}\right|,\left|x_{2}\right|<\mu} d x_{1} d x_{2}\left|\frac{1}{r^{2}} \iint_{x_{1}^{2}+x_{2}^{2}<2 r^{2}} u d x_{1} d x_{2}\right|^{\frac{10}{3}} \\
& \leq C\left(\frac{\mu}{r}\right)^{2} \int_{B(\sqrt{3} r)}|u|^{\frac{10}{3}} d x .
\end{aligned}
$$

On the other hand, by Hölder's inequality, we have

$$
\begin{aligned}
& \int_{B(\mu)}\left|u-\overline{u_{\sqrt{2} r}^{h}}\right|^{\frac{10}{3}} d x \\
\leq & \iiint_{\left|x_{1}\right|,\left|x_{2}\right|,\left|x_{3}\right|<\mu}\left|u-\overline{u_{\sqrt{2} r}^{h}}\right|^{\frac{10}{3}} d x \\
\leq & \left\|\left|u-\overline{u_{\sqrt{2} r}^{h}}\right|^{\frac{4}{3}}\right\|_{L^{\frac{5}{2}}\left(\left|x_{1}\right|,\left|x_{2}\right|,\left|x_{3}\right|<\mu\right)}\|\| u-\overline{u_{\sqrt{2} r}^{h}}\left\|_{L^{10}\left(\left|x_{3}\right|<\mu\right)}\right\|_{L^{2}\left(\left|x_{1}\right|,\left|x_{2}\right|<\mu\right)} \\
& \times\|\| u-\overline{u_{\sqrt{2} r}^{h}}\left\|_{L^{2}\left(\left|x_{3}\right|<\mu\right)}\right\|_{L^{10}\left(\left|x_{1}\right|,\left|x_{2}\right|<\mu\right)} \\
\leq & \|\| u-\overline{u_{\sqrt{2} r}^{h}}\left\|_{L^{10}\left(\left|x_{3}\right|<\mu\right)}\right\|_{L^{2}\left(\left|x_{1}\right|,\left|x_{2}\right|<\mu\right)}^{\frac{5}{3}}\|\| u-\overline{u_{\sqrt{2} r}^{h}}\left\|_{L^{2}\left(\left|x_{3}\right|<\mu\right)}\right\|_{L^{10}\left(\left|x_{1}\right|,\left|x_{2}\right|<\mu\right)}^{\frac{5}{3}} .
\end{aligned}
$$


In the light of the Gagliardo-Nirenberg inequality [13] on the bounded domain in $\mathbb{R}$, we know that

$$
\left\|f\left(x_{3}\right)\right\|_{L^{10}\left(\left|x_{3}\right|<\mu\right)} \leq C\left\|f\left(x_{3}\right)\right\|_{L^{2}\left(\left|x_{3}\right|<\mu\right)}^{\frac{3}{5}}\left\|\partial_{3} f\left(x_{3}\right)\right\|_{L^{2}\left(\left|x_{3}\right|<\mu\right)}^{\frac{2}{5}}+C \mu^{-\frac{2}{5}}\left\|f\left(x_{3}\right)\right\|_{L^{2}\left(\left|x_{3}\right|<\mu\right)}
$$

for any $f \in W^{1,2}(-\mu, \mu)$.

With the help of this inequality and Hölder's inequality, we easily get

$$
\begin{aligned}
& \|\| u-\overline{u_{\sqrt{2} r}^{h}}\left\|_{L^{10}\left(\left|x_{3}\right|<\mu\right)}\right\|_{L^{2}\left(\left|x_{1}\right|,\left|x_{2}\right|<\mu\right)} \\
& \leq C\|\| u-\overline{u_{\sqrt{2} r}^{h}}\left\|_{L^{2}\left(\left|x_{3}\right|<\mu\right)}^{\frac{3}{5}}\right\| \partial_{3} u-\partial_{3} \overline{u_{\sqrt{2} r}^{h}}\left\|_{L^{2}\left(\left|x_{3}\right|<\mu\right)}^{\frac{2}{5}}\right\|_{L^{2}\left(\left|x_{1}\right|,\left|x_{2}\right|<\mu\right)} \\
& +C \mu^{-\frac{2}{5}}\|\| u-\overline{u_{\sqrt{2} r}^{h}}\left\|_{L^{2}\left(\left|x_{3}\right|<\mu\right)}\right\|_{L^{2}\left(\left|x_{1}\right|,\left|x_{2}\right|<\mu\right)} \\
& \leq C\left\|u-\overline{u_{\sqrt{2} r}^{h}}\right\|_{L^{2}\left(\left|x_{1}\right|,\left|x_{2}\right|,\left|x_{3}\right|<\mu\right)}^{\frac{3}{5}}\left\|\partial_{3} u-\partial_{3} \overline{u_{\sqrt{2} r}^{h}}\right\|_{L^{2}\left(x_{1}|,| x_{2}|,| x_{3} \mid<\mu\right)}^{\frac{2}{5}} \\
& +C \mu^{-\frac{2}{5}}\left\|u-\overline{u_{\sqrt{2} r}^{h}}\right\|_{L^{2}\left(\left|x_{1}\right|,\left|x_{2}\right|,\left|x_{3}\right|<\mu\right)} \\
& =C\|\| u-\overline{u_{\sqrt{2} r}^{h}}\left\|_{L^{2}\left(\left|x_{1}\right|,\left|x_{2}\right|<\mu\right)}\right\|_{L^{2}\left(\left|x_{3}\right|<\mu\right)}^{\frac{3}{5}}\left\|\partial_{3} u-\partial_{3} \overline{u_{\sqrt{2} r}^{h}}\right\|_{L^{2}\left(\left|x_{1}\right|,\left|x_{2}\right|<\mu\right)} \|_{L^{2}\left(\left|x_{3}\right|<\mu\right)}^{\frac{2}{5}} \\
& +C \mu^{-\frac{2}{5}}\|\| u-\overline{u_{\sqrt{2} r}^{h}}\left\|_{L^{2}\left(\left|x_{1}\right|,\left|x_{2}\right|<\mu\right)}\right\|_{L^{2}\left(\left|x_{3}\right|<\mu\right)} \\
& \leq C\|\| u\left\|_{L^{2}\left(x_{1}^{2}+x_{2}^{2}<2 r^{2}\right)}\right\|_{L^{2}\left(\left|x_{3}\right|<\mu\right)}^{\frac{3}{5}}\|\| \partial_{3} u\left\|_{L^{2}\left(x_{1}^{2}+x_{2}^{2}<2 r^{2}\right)}\right\|_{L^{2}\left(\left|x_{3}\right|<\mu\right)}^{\frac{2}{5}} \\
& +C \mu^{-\frac{2}{5}}\|\| u\left\|_{L^{2}\left(x_{1}^{2}+x_{2}^{2}<2 r^{2}\right)}\right\|_{L^{2}\left(\left|x_{3}\right|<\mu\right)} \\
& \leq C\|u\|_{L^{2}(B(\sqrt{3} r))}^{\frac{3}{5}}\left\|\partial_{3} u\right\|_{L^{2}(B(\sqrt{3} r))}^{\frac{2}{5}}+C \mu^{-\frac{2}{5}}\|u\|_{L^{2}(B(\sqrt{3} r))},
\end{aligned}
$$

where we have used the fact that $\left\|u-\overline{u_{\sqrt{2} r}^{h}}\right\|_{L^{2}\left(x_{1}^{2}+x_{2}^{2}<2 r^{2}\right)} \leq 2\|u\|_{L^{2}\left(x_{1}^{2}+x_{2}^{2}<2 r^{2}\right)}$.

In addition, taking advantage of Minkowski's inequality and Poincáre-Sobolev's inequality on the ball in $\mathbb{R}^{2}$, we find that

$$
\begin{aligned}
\|\| u-\overline{u_{\sqrt{2} r}^{h}}\left\|_{L^{2}\left(\left|x_{3}\right|<\mu\right)}\right\|_{L^{10}\left(\left|x_{1}\right|,\left|x_{2}\right|<\mu\right)} & \leq\|\| u-\overline{u_{\sqrt{2} r}^{h}}\left\|_{L^{10}\left(x_{1}^{2}+x_{2}^{2}<2 r^{2}\right)}\right\|_{L^{2}\left(\left|x_{3}\right|<\mu\right)} \\
& \leq C\|\| u\left\|_{L^{2}\left(x_{1}^{2}+x_{2}^{2}<2 r^{2}\right)}^{\frac{1}{5}}\right\| \nabla_{h} u\left\|_{L^{2}\left(x_{1}^{2}+x_{2}^{2}<2 r^{2}\right)}^{\frac{4}{5}}\right\|_{L^{2}\left(\left|x_{3}\right|<\mu\right)} \\
& \leq C\|u\|_{L^{2}(B(\sqrt{3} r))}^{\frac{1}{5}}\left\|\nabla_{h} u\right\|_{L^{2}(B(\sqrt{3} r))}^{\frac{4}{5}} .
\end{aligned}
$$

Plugging these two estimates into (2.15), we infer that

$$
\begin{aligned}
& \int_{B(\mu)}\left|u-\overline{u_{\sqrt{2} r}^{h}}\right|^{\frac{10}{3}} d x \\
\leq & C\|u\|_{L^{2}(B(\sqrt{3} r))}^{\frac{4}{3}}\left\|\partial_{3} u\right\|_{L^{2}(B(\sqrt{3} r))}^{\frac{2}{3}}\left\|\nabla_{h} u\right\|_{L^{2}(B(\sqrt{3} r))}^{\frac{4}{3}}+C \mu^{-\frac{2}{3}}\|u\|_{L^{2}(B(\sqrt{3} r))}^{2}\left\|\nabla_{h} u\right\|_{L^{2}(B(\sqrt{3} r))}^{\frac{4}{3}},
\end{aligned}
$$

which together with (2.13) and (2.14) implies

$$
\begin{aligned}
& \int_{B(\mu)}|u|^{\frac{10}{3}} d x \leq C\|u\|_{L^{2}(B(\sqrt{3} r))}^{\frac{4}{3}}\left\|\partial_{3} u\right\|_{L^{2}(B(\sqrt{3} r))}^{\frac{2}{3}}\left\|\nabla_{h} u\right\|_{L^{2}(B(\sqrt{3} r))}^{\frac{4}{3}} \\
& +C \mu^{-\frac{2}{3}}\|u\|_{L^{2}(B(\sqrt{3} r))}^{2}\left\|\nabla_{h} u\right\|_{L^{2}(B(\sqrt{3} r))}^{\frac{4}{3}}+C\left(\frac{\mu}{r}\right)^{2} \int_{B(\sqrt{3} r)}|u|^{\frac{10}{3}} d x .
\end{aligned}
$$


Integrating this inequality with respect to $t$ on $\left(-\mu^{2}, 0\right)$ and utilizing Hölder's inequality yields that

$$
\begin{aligned}
& \iint_{Q(\mu)}|u|^{\frac{10}{3}} d x d s \\
\leq & C\left(\sup _{-r^{2} \leq s \leq 0} \int_{B(\sqrt{3} r)}|u|^{2} d x\right)^{\frac{2}{3}}\left(\iint_{Q(\sqrt{3} r)}\left|\partial_{3} u\right|^{2} d x d s\right)^{\frac{1}{3}}\left(\iint_{Q(\sqrt{3} r)}\left|\nabla_{h} u\right|^{2} d x d s\right)^{\frac{2}{3}} \\
& +C\left(\sup _{-r^{2} \leq s \leq 0} \int_{B(\sqrt{3} r)}|u|^{2} d x\right)\left(\iint_{Q(\sqrt{3} r)}\left|\nabla_{h} u\right|^{2} d x d s\right)^{\frac{2}{3}} \\
& +C\left(\frac{\mu}{r}\right)^{2} \iint_{Q(\sqrt{3} r)}|u|^{\frac{10}{3}} d x d s,
\end{aligned}
$$

which in turn implies the desired estimate.

\section{Proofs of Theorem 1.1 and 1.2}

In the spirit of [20], Theorem 1.1 and 1.2 turn out to be the corollaries of the following propositions.

Proposition 3.1. Assume that the pair $(u, \Pi)$ is a suitable weak solution to the NavierStokes equations. For any $M>0$, there exists a positive constant $\varepsilon(M)$ such that if

$$
r^{2-\frac{3}{p}-\frac{2}{q}}\left(\int_{-r^{2}}^{0}\left(\int_{B(r)}|\nabla u|^{p} d x\right)^{\frac{q}{p}} d s\right)^{\frac{1}{q}} \leq M,
$$

and

$$
r_{*}^{2-\frac{3}{p}-\frac{2}{q}}\left(\int_{-r_{*}^{2}}^{0}\left(\int_{B\left(r_{*}\right)}\left|\omega_{h}\right|^{p} d x\right)^{\frac{q}{p}} d s\right)^{\frac{1}{q}} \leq \varepsilon,
$$

for some $p, q$ with $2 \leq 3 / p+2 / q<3,1<p, q \leq \infty$ and some $r_{*} \in\left(0, \min \left\{1 / 2,\left(E_{3}(u, 1)+\right.\right.\right.$ $\left.\left.\left.P_{3 / 2}(u, 1)\right)^{-2}\right\}\right]$, then $(0,0)$ is regular point.

Proof. Assume that the statement fails, then there exists a sequence $\left(u^{k}, \Pi^{k}\right)$ of the suitable weak solutions to Navier-Stokes equations such that

$$
E_{*, p, q}\left(u^{k}, r\right) \leq M
$$

for all $0<r \leq 1$ and

$$
W_{p, q}\left(\omega_{h}^{k}, r_{k}\right) \leq \frac{1}{k}
$$

for $r_{k} \in\left(0, \min \left\{1 / 2,\left(E_{3}(u, 1)+P_{3 / 2}(u, 1)\right)^{-2}\right\}\right]$ and $(0,0)$ is a singular point of $u^{k}$. Therefore, in light of Theorem 2.2, there exists an absolute constant $\varepsilon_{0}>0$ such that

$$
E_{3}\left(u^{k}, r\right)+P_{3 / 2}\left(\Pi^{k}, r\right) \geq \varepsilon_{0},
$$

for any $0<r \leq r_{k}$. With the help of Lemma 2.1 and (3.2), we can deduce that

$$
E\left(u^{k}, r\right)+E_{*}\left(u^{k}, r\right)+P_{3 / 2}\left(\Pi^{k}, r\right) \leq C(M),
$$


for all $0<r \leq r_{k}$.

Set $v^{k}(x, t)=r_{k} u^{k}\left(r_{k} x, r_{k}^{2} t\right), q^{k}(x, t)=r_{k}^{2} \Pi^{k}\left(r_{k} x, r_{k}^{2} t\right), \widetilde{\omega}^{k}(x, t)=r_{k}^{2} \omega^{k}\left(r_{k} x, r_{k}^{2} t\right)$, where $\omega^{k}=\operatorname{curl} u^{k}$. For any $R>0$, a straightforward computation gives

$$
\begin{array}{lc}
E\left(v^{k}, R\right)=E\left(u^{k}, r_{k} R\right), & E_{3}\left(v^{k}, R\right)=E_{3}\left(u^{k}, r_{k} R\right), \\
P_{3 / 2}\left(q^{k}, R\right)=P_{3 / 2}\left(\Pi^{k}, r_{k} R\right), & W_{p, q}\left(\widetilde{\omega}_{h}^{k}, r\right)=W_{p, q}\left(\omega_{h}^{k}, r_{k} r\right) .
\end{array}
$$

This implies that, for all $0<r \leq 1$ and $k \in \mathbb{N}$,

$$
\begin{aligned}
& E\left(v^{k}, r\right)+E_{3}\left(v^{k}, r\right)+P_{3 / 2}\left(q^{k}, r\right) \leq C(M), \\
& W_{p, q}\left(\widetilde{\omega}_{h}^{k}, 1\right) \leq \frac{1}{k} \\
& E_{3}\left(v^{k}, r\right)+P_{3 / 2}\left(q^{k}, r\right) \geq \varepsilon_{0} .
\end{aligned}
$$

It is obvious to see that the pair $\left(v^{k}, q^{k}\right)$ solves the following system

$$
\left\{\begin{array}{l}
v_{t}^{k}+v^{k} \cdot \nabla v^{k}-\Delta v^{k}+\nabla q^{k}=0 \\
\operatorname{div} v^{k}=0
\end{array}\right.
$$

in the sense of distribution on $Q(1)$. For any text function $\phi \in L^{3}\left(-1,0 ; W_{0}^{2,2}(B(1))\right)$, simple computations give that

$$
\begin{gathered}
\iint_{Q(1)}\left(v^{k} \otimes v^{k}\right): \nabla \phi d x d t \leq\left\|v^{k}\right\|_{L^{\infty}\left(L^{2}\right)}\left\|v^{k}\right\|_{L^{2}\left(L^{6}\right)}\|\nabla \phi\|_{L^{2}\left(L^{3}\right)} \leq C(M)\|\phi\|_{L^{3}\left(W_{0}^{2,2}\right)}, \\
\iint_{Q(1)}\left|\nabla v^{k}\right||\nabla \phi| d x d t \leq\left\|\nabla v^{k}\right\|_{L^{2}\left(L^{2}\right)}\|\nabla \phi\|_{L^{2}\left(L^{2}\right)} \leq C(M)\|\phi\|_{L^{3}\left(W_{0}^{2,2}\right)}, \\
\iint_{Q(1)} q^{k} \operatorname{div} \phi d x d t \leq\left\|q^{k}\right\|_{L^{3 / 2}\left(L^{3 / 2}\right)}\|\phi\|_{L^{3}\left(L^{3}\right)} \leq C(M)\|\phi\|_{L^{3}\left(W_{0}^{2,2}\right)},
\end{gathered}
$$

which implies $\partial_{t} v^{k} \in L^{\frac{3}{2}}\left(-1,0 ;\left(W_{0}^{2,2}(B(1))\right)^{*}\right)$, where $\left(W_{0}^{2,2}(B(1))\right)^{*}$ denotes the dual space of $W_{0}^{2,2}(B(1))$. This together with the fact that $\nabla v^{k} \in L^{2} L^{2}(Q(1))$ allows us to obtain a subsequence (still denoted by $k$ ) by using Aubin-Lions Lemma ([22, Theorem 2.1, p.184]) such that

$$
v^{k} \rightarrow v \quad \text { in } L^{2}(Q(1)) .
$$

By means of the interpolation inequality, it follows from $v^{k} \in L^{\infty}\left(-1,0 ; L^{2}(B(1))\right) \cap$ $L^{2}\left(-1,0 ; W^{1,2}(B(1))\right)$ that $v^{k} \in L^{10 / 3}(Q(1))$. Thus, by a diagonalization process, we find that

$$
\begin{aligned}
& v^{k} \rightarrow v \quad \text { in } \quad L^{3}(Q(1)), \\
& \omega_{h}^{k} \rightarrow 0 \quad \text { in } \quad L^{p} L^{q}(Q(1)), \\
& q^{k} \rightarrow q \quad \text { in } \quad L^{3 / 2}(Q(1)) .
\end{aligned}
$$

Furthermore, $v=\left(v_{1}, v_{2}, v_{3}\right)$ and $q$ solve

$$
\left\{\begin{array}{l}
v_{t}+v \cdot \nabla v-\Delta v+\nabla q=0 \\
\operatorname{div} v=0, \omega_{h}=0
\end{array}\right.
$$


in the sense of distribution. Form Lemma A.2 in Appendix or Chae, Kang and Lee's main result in [4, we know that there exist a constant $0<r^{\prime} \leq 1$ such that

$$
|v(x, t)| \leq C(M), \quad(x, t) \in Q\left(r^{\prime}\right) .
$$

Notice that (3.6) is also true for the convergence subsequence obtained in the (3.8)-(3.10). Based on this, we can pass to the limit in (3.6) to arrive at

$$
E_{3}(v, r)+\lim _{k \rightarrow \infty} P_{3 / 2}\left(q^{k}, r\right) \geq \varepsilon_{0} .
$$

Employing Lemma 2.3 for $q=3 / 2$ and any $0<2 r<\sqrt{r} \leq \min \left\{1 / 4, r^{\prime}\right\}$, we can get

$$
P_{3 / 2}\left(q^{n_{k}}, r\right) \leq C\left(\frac{\sqrt{r}}{r}\right)^{2} E_{3}\left(v^{k}, \sqrt{r}\right)+C\left(\frac{r}{\sqrt{r}}\right) P_{3 / 2}\left(q^{k}, \sqrt{r}\right) \leq C \sqrt{r}
$$

where we have used (3.4) and (3.12). This estimate together with (3.13) yields that

$$
C r^{3}+C \sqrt{r} \geq \varepsilon_{0},
$$

for all $0<r<\min \left\{\frac{1}{8}, \frac{1}{2} r^{\prime}\right\}$. This will lead to a contradiction when $r$ is sufficiently small, which concludes the proof of this proposition.

Neustupa and Penel's result in [12] ensures the interior regularity of system (1.16). With the help of this fact together with Lemma 2.1, it is not hard to show the following proposition by modifying slightly the above proof. We omit the detail here.

Proposition 3.2. Assume that the pair $(u, \Pi)$ is a suitable weak solution to the NavierStokes equations. For any $M>0$, there exists a positive constant $\varepsilon(M)$ such that if

$$
\sup _{0<r \leq 1} r^{1-\frac{3}{p}-\frac{2}{q}}\left(\int_{-r^{2}}^{0}\left(\int_{B(r)}|u|^{p} d x\right)^{\frac{q}{p}} d s\right)^{\frac{1}{q}} \leq M,
$$

and

$$
r_{*}^{1-\frac{3}{p}-\frac{2}{q}}\left(\int_{-r_{*}^{2}}^{0}\left(\int_{B\left(r_{*}\right)}\left|u_{3}\right|^{p} d x\right)^{\frac{q}{p}} d s\right)^{\frac{1}{q}} \leq \varepsilon,
$$

for $p, q$ satisfying $1 \leq 3 / p+2 / q<2,1<p, q \leq \infty$, and some $r_{*} \in\left(0, \min \left\{\frac{1}{2},\left(E_{3}(u, 1)+\right.\right.\right.$ $\left.\left.\left.P_{3 / 2}(u, 1)\right)^{-2}\right\}\right]$, then $(0,0)$ is regular point.

Now we are in a position to complete the proofs of Theorem 1.1 and 1.2 .

Proof of Theorem 1.1. It follows from (1.10) and Lemma 2.4 that there is a constant $r_{*}$ such that

$$
E_{*, p^{\prime}, q^{\prime}}(u, r) \leq C(M), \forall 0<r \leq r_{*} .
$$

Set $v(x, t)=r_{*} u\left(r_{*} x, r_{*}^{2} t\right), q(x, t)=r_{*}^{2} p\left(r_{*} x, r_{*}^{2} t\right)$ and $\widetilde{\omega}=r_{*}^{2} \omega\left(r_{*} x, r_{*}^{2} t\right)$, where $\omega=\operatorname{curl} u$. Therefore, for any $0<r \leq 1, E_{*, p^{\prime}, q^{\prime}}(v, r)=E_{*, p^{\prime}, q^{\prime}}\left(u, r r_{*}\right) \leq C(M)$. Moreover, we also have

$$
\liminf _{r \rightarrow 0} W_{p, q}(\widetilde{\omega}, r)=\liminf _{r \rightarrow 0} W_{p, q}\left(\omega, r r_{*}\right)=\liminf _{r r_{*} \rightarrow 0} W_{p, q}\left(\omega, r r_{*}\right)<\varepsilon_{11} .
$$

From this inequalities and the definition of the limit inferior, we deduce that the suitable weak solution $(v, q)$ satisfies the condition of Proposition 3.1. Hence, $(0,0)$ is a regular point of $v$, which means that $(0,0)$ is also regular point of $u$. Therefore, Theorem 1.1 is proved. 
Proof of Theorem 1.2. (1) By the condition (1.13), we find that there exists a constant $r_{*}>0$ such that

$$
\sup _{0<r \leq r_{*}} E_{p, q}(u, r) \leq M .
$$

Set $v(x, t)=r_{*} u\left(r_{*} x, r_{*}^{2} t\right), q(x, t)=r_{*}^{2} p\left(r_{*} x, r_{*}^{2} t\right)$. It is clear that, for any $0<r \leq 1$,

$$
E_{p, q}(v, r)=E_{p, q}\left(u, r r_{*}\right) \leq M
$$

and

$$
\liminf _{r \rightarrow 0} E_{p, q}(v, r)=\liminf _{r \rightarrow 0} E_{p, q}\left(u, r r_{*}\right)=\liminf _{r r_{*} \rightarrow 0} E_{p, q}\left(u, r r_{*}\right)<\varepsilon_{21} .
$$

Now we can employ Proposition 3.2 to obtain that $(0,0)$ is a regular point of $v$, which in turn implies that $(0,0)$ is also a regular point of $u$. Therefore, we finish the proof of the first part of this theorem.

(2) By Hölder's inequality, without loss of generality, we just deal with the case $3 / p+2 / q=$ 3. Thanks to our assumption (1.14) and Lemma 2.1, we see that

$$
E(u, r)+E_{*}(u, r)+P_{3 / 2}(p, r) \leq C(M),
$$

for any $0<r \leq \min \left\{\frac{1}{4},\left(E_{3}(u, 1)+P_{3 / 2}(u, 1)\right)^{-2}\right\}$, which yields that $E_{3}(u, r) \leq C(M)$.

Using the triangle inequality, Hölder's inequality, Sobolev-Poincáre's inequality and Gagliardo-Nirenberg's inequality, one can deduce that

$$
\begin{aligned}
E_{3}\left(u_{3}, \mu\right) & \leq C E_{3}\left(u_{3}-\overline{u_{3}} r^{\prime \prime}, \mu\right)+C\left(\frac{\mu}{r^{\prime \prime}}\right) E_{3}\left(u_{3}, r^{\prime \prime}\right) \\
& \leq C\left(\frac{r^{\prime \prime}}{\mu}\right)^{2} E^{\frac{1}{q}}\left(u_{3}, r^{\prime \prime}\right) E_{*}^{1-\frac{1}{q}}\left(u_{3}, r^{\prime \prime}\right) E_{* ; p, q}\left(u_{3}, r^{\prime \prime}\right)+C\left(\frac{\mu}{r^{\prime \prime}}\right) E_{3}\left(u_{3}, r^{\prime \prime}\right),
\end{aligned}
$$

where $0<\mu \leq r^{\prime \prime}$ and $\overline{u_{3}} r^{\prime \prime}=\frac{1}{\left|B\left(r^{\prime \prime}\right)\right|} \int_{B\left(r^{\prime \prime}\right)} u_{3} d x$. For detailed computation, we refer the reader to [7, Lemma 3.2, 3.3 and 3.5, p.168-171].

It follows from (3.15) and (3.16) that

$$
E_{3}\left(u_{3}, \mu\right) \leq C(M)\left(\frac{r^{\prime \prime}}{\mu}\right)^{2} \varepsilon_{22}+C\left(\frac{\mu}{r^{\prime \prime}}\right),
$$

where $r^{\prime \prime} \leq \min \left\{\frac{1}{4},\left(E_{3}(u, 1)+P_{3 / 2}(u, 1)\right)^{-2}\right\}$. Fix $r^{\prime \prime}$, then choose $\mu^{\prime}$ and $\varepsilon$ such that $E_{3}\left(u_{3}, r\right) \leq \varepsilon$, for $r \leq \mu^{\prime}$. Now we can make use of the result in the first part of Theorem 1.2 to finish the proof of the second part.

\section{Proof of Theorem 1.3}

Proof of Theorem 1.3. The condition (1.18) guarantees that there is a constant $r_{1}$ such that

$$
E_{*, h}(u, r) \leq \varepsilon_{31}, \text { for any } \quad 0<r \leq r_{1} .
$$

According to the regularization condition (1.4) (or condition (1.5)), it is enough to show that there exists a constant $0<r_{2} \leq r_{1}$ such that

$$
E_{10 / 3}(u, r) \leq \varepsilon_{2}, \text { for any } \quad 0<r \leq r_{2}
$$


By the fundamental property of the usual nonnegative cut-off function, it follows from the local energy inequality (1.2) that

$$
E(u, r)+E_{*}(u, r) \leq C\left[E_{2}(u, 2 r)+E_{3}(u, 2 r)+E_{3}^{1 / 3}(u, 2 r) P_{3 / 2}^{2 / 3}(p, 2 r)\right],
$$

which in turn implies that

$$
\begin{aligned}
& \varepsilon_{31}^{1 / 9} E(u, r)+\varepsilon_{31}^{1 / 9} E_{*}(u, r) \\
\leq & C \varepsilon_{31}^{1 / 9}\left(E_{10 / 3}(u, 2 r)\right)^{3 / 5}+C \varepsilon_{31}^{1 / 9}\left(E_{10 / 3}(u, 2 r)\right)^{9 / 10} \\
& +C \varepsilon_{31}^{1 / 9}\left(E_{3 / 10}(u, 2 r)\right)^{10 / 3}\left(P_{5 / 3}(p, 2 r)\right)^{3 / 5} \\
\leq & C E_{10 / 3}(u, 2 r)+C \varepsilon_{31}^{5 / 18}+C \varepsilon_{31}+\varepsilon_{31}^{10 / 63}\left(P_{5 / 3}(p, 2 r)\right)^{6 / 7} \\
\leq & C_{1} E_{10 / 3}(u, 2 r)+\varepsilon_{31}^{1 / 18}\left(\frac{\rho}{r}\right)^{5 / 3} \varepsilon^{1 / 9} P_{5 / 3}(p, \rho)+C_{3} \varepsilon_{31}^{1 / 9},
\end{aligned}
$$

where we have used Hölder's and Young's inequalities and the fact that

$$
P_{5 / 3}(p, 2 r) \leq\left(\frac{\rho}{r}\right)^{5 / 3} P_{5 / 3}(p, \rho) \text { for } 2 r \leq \rho \leq r_{1} .
$$

By Lemma 2.3 with $q=5 / 3$, we get

$$
\varepsilon_{31}^{1 / 9} P_{5 / 3}(p, 2 r) \leq \varepsilon_{31}^{1 / 9} C\left(\frac{\rho}{r}\right)^{5 / 3} E_{10 / 3}(\rho)+C\left(\frac{r}{\rho}\right)^{4 / 3} \varepsilon_{31}^{1 / 9} P_{5 / 3}(\rho) .
$$

Set

$$
\psi(r)=E_{10 / 3}(u, r)+\varepsilon_{31}^{1 / 9} E(u, r)+\varepsilon_{31}^{1 / 9} E_{*}(u, r)+\varepsilon_{31}^{1 / 9} P_{5 / 3}(p, r) .
$$

Thanks to Lemma 2.5, we arrive at

$$
E_{10 / 3}(u, r) \leq C\left(\frac{\rho}{\mu}\right)^{5 / 3} \psi(\rho) \varepsilon_{31}^{5 / 9}+C\left(\frac{\mu}{\rho}\right)^{1 / 3} E_{10 / 3}(u, \rho),
$$

which in turn implies that

$$
\begin{aligned}
\psi(r) \leq & C\left(\frac{\rho}{r}\right)^{5 / 3} \psi(\rho) \varepsilon_{31}^{5 / 9}+C\left(\frac{r}{\rho}\right)^{1 / 3} E_{10 / 3}(u, \rho)+\varepsilon_{31}^{1 / 18}\left(\frac{\rho}{r}\right)^{5 / 3} \varepsilon_{31}^{1 / 9} P_{5 / 3}(p, \rho)+C_{3} \varepsilon_{31}^{1 / 9} \\
& +\varepsilon_{31}^{1 / 9} C\left(\frac{\rho}{r}\right)^{2} E_{10 / 3}(u, \rho)+C\left(\frac{r}{\rho}\right) \varepsilon_{31}^{1 / 9} P_{5 / 3}(p, \rho) \\
\leq & C \varepsilon_{31}^{1 / 18}\left(\frac{\rho}{r}\right)^{5 / 3} \psi(\rho)+C\left(\frac{r}{\rho}\right)^{1 / 3} \psi(\rho)+\varepsilon_{31}^{1 / 9} C \\
\leq & C_{1} \lambda^{-5 / 3} \varepsilon_{31}^{1 / 18} \psi(\rho)+C_{2} \lambda^{1 / 3} \psi(\rho)+\varepsilon_{31}^{1 / 9} C_{3}
\end{aligned}
$$

where $\lambda=\mu / \rho \leq 1 / 4$. Choosing $\lambda, \varepsilon_{31}$ such that

$$
q=2 C_{2} \lambda^{1 / 3}<1 \quad \text { and } \quad \varepsilon_{31}=\min \left\{\left(\frac{q \lambda^{5 / 3}}{2 C_{1}}\right)^{18},\left(\frac{(1-q) \lambda^{10 / 3} \varepsilon_{2}}{2 C_{3}}\right)^{9}\right\} .
$$

It follows from (4.1) that

$$
\psi(\lambda \rho) \leq q \psi(\rho)+\varepsilon_{31}^{1 / 9} C_{3} .
$$


Iterating this inequality, we see that

$$
\psi\left(\lambda^{k} \rho\right) \leq q^{k} \psi(\rho)+\frac{1}{2} \lambda^{5 / 3} \varepsilon_{2} .
$$

By the definition of $\psi(r)$, we know that there exists a positive number $K_{0}$ such that

$$
q^{K_{0}} \psi\left(r_{1}\right) \leq 4 \frac{C\left(\left(\|u\|_{L^{\infty} L^{2}},\|u\|_{\left.\left.L^{2} W^{1,2},\|p\|_{L^{5 / 3} L^{5 / 3}}\right)\right)}\right.\right.}{r_{1}^{5 / 3}} q^{K_{0}} \leq \frac{1}{2} \lambda^{5 / 3} \varepsilon_{2} .
$$

Let $r_{2}=\lambda^{K_{0}} r_{1}$. Therefore, for all $0<r \leq r_{2}$, there exists a constant $k \geq K_{0}$ such that $\lambda^{k+1} r_{1} \leq r \leq \lambda^{k} r_{1}$. Straightforward calculations show that

$$
\begin{aligned}
E_{10 / 3}(u, r) & =\frac{1}{r^{5 / 3}} \iint_{Q(r)}|u|^{10 / 3} d x d t \\
& \leq \frac{1}{\lambda^{k+1} r_{1}} \iint_{Q\left(\lambda^{k} r_{1}\right)}|u|^{10 / 3} d x d t \\
& \leq \frac{1}{\lambda^{5 / 3}} \psi\left(\lambda^{k} r_{1}\right) \\
& \leq \frac{1}{\lambda^{5 / 3}}\left(q^{k-K_{0}} q^{K_{0}} \psi\left(r_{1}\right)+\frac{1}{2} \lambda^{5 / 3} \varepsilon_{2}\right) \\
& \leq \varepsilon_{2},
\end{aligned}
$$

which ends the proof of this theorem.

\section{A Appendix}

Lemma A.1. Let $k(x, t)$ denote the fundamental solution of heat equation with $k(x, t)=0$ for $t \leq 0$, and $f \in L^{q^{\prime}}\left(0, T ; L^{q}(\Omega)\right)$, and set

$$
k * f(x, t)=\int_{0}^{T} \int_{\Omega} k(x-y, t-s) f(y, s) d y d s .
$$

Then

$$
\|k * f\|_{L^{r^{\prime}\left(0, T ; L^{r}(\Omega)\right)}} \leq C\|f\|_{L^{q^{\prime}\left(0, T ; L^{q}(\Omega)\right)}}
$$

with

$$
n\left(\frac{1}{q}-\frac{1}{r}\right)+2\left(\frac{1}{q^{\prime}}-\frac{1}{r^{\prime}}\right)<2
$$

Proof. Since the proof is standard, we omit it (see, for example, [21]).

Lemma A.2. Let the pair $(v, \pi)$ be the weak solution to the following system

$$
\left\{\begin{array}{l}
v_{t}-\Delta v+v \cdot \nabla v+\nabla \pi=0 \\
\operatorname{div} v=0, \omega_{h}=0
\end{array}\right.
$$

where $\omega=$ curl $v$. Then one has $v \in L_{l o c}^{\infty}$. 
Proof. The proof relies on a bootstrapping argument. Since $\omega_{h}=0$ and $\operatorname{div} \omega=0$, the equations of vorticity read as

$$
\omega_{3 t}-\Delta \omega_{3}+v \cdot \nabla \omega_{3}=\omega_{3} \partial_{3} v_{3}, \partial_{3} \omega_{3}=0 .
$$

By means of $\partial_{3} \omega_{3}=0$, we can control the stretching term $\omega_{3} \partial_{3} v_{3}$ just as the term $v \cdot \nabla \omega_{3}$ when we apply the standard energy method to get $\omega_{3} \in L^{\infty} L^{2} \cap L^{2}\left(W^{1,2}\right)$ under the condition $v \in L^{\infty} L^{2} \cap L^{2}\left(W^{1,2}\right)$.

Thanks to $v \cdot \nabla v=\frac{1}{2} \nabla v^{2}+\omega \times v$, the original system can be rewritten as

$$
\left\{\begin{array}{l}
v_{t}-\Delta v+\nabla \Pi=-\omega \times v=-\left(-v_{2} \omega_{3}, v_{1} \omega_{3}, 0\right) \\
\operatorname{div} v=0, \quad \Pi=\pi+\frac{1}{2} v^{2}
\end{array}\right.
$$

Notice that if we assume that $u \in L_{t, x}^{p_{k}}, k=1,2, \cdots$, then $\omega \times v \in L^{\frac{10 p_{k}}{3 p_{k}+10}}$. It follows from the classical interior estimate for the Stokes system that $\nabla \Pi \in L^{\frac{10 p_{k}}{3 p_{k}+10}}$. Therefore $v_{t}-\Delta v=$ $-\omega \times v-\nabla \Pi \in L^{\frac{10 p_{k}}{3 p_{k}+10}}$. Notice also that $v=k *(-\omega \times v-\nabla \Pi)+H(x, t)=: v^{1}+H(x, t)$, where $H(x, t)$ is the solution of the heat equations. At first, it is clear that $H(x, t) \in L^{\infty}$. Secondly, by Lemma A.1, we can get $v^{1} \in L^{p_{k+1}}$ where $p_{k+1}$ satisfies

$$
3\left(\frac{3}{10}+\frac{1}{p_{k}}-\frac{1}{p_{k+1}}\right)+2\left(\frac{3}{10}+\frac{1}{p_{k}}-\frac{1}{p_{k+1}}\right)<2,
$$

namely,

$$
\frac{1}{p_{k}}-\frac{1}{p_{k+1}}<\frac{1}{10}
$$

Set $\frac{1}{p_{k}}-\frac{1}{p_{k+1}}=\frac{1}{20}$, and recall that $p_{1}=10 / 3$. Then, after a finite number of bootstrapping steps, we finally obtain $v \in L_{t, x}^{\infty}$.

Remark A.1. After this paper was submitted for publication, we learnt that the regular condition (1.8) was improved by Wang, Zhang and Zhang in the preprint [25]. We would like to point out that our Theorem 1.2 is different from Theorem 1.2 in [25]. Especially, the fist result of Theorem 1.2 does not seem to be comparable to that of [25]. The first author express his thank to Professor Zhifei Zhang for providing the paper [26].

Acknowledgements: The second author is supported in part by the National Natural Science Foundation of China under grant No.11101405 and the President Fund of UCAS.

\section{References}

[1] J. Beale, T. Kato and A. Majda, Remarks on the breakdown of smooth solutions for the 3-D Euler equations. Comm. Math. Phys. 94 (1984), 61-66.

[2] L. Caffarelli, R. Kohn and L. Nirenberg, Partial regularity of suitable weak solutions of NavierStokes equation, Comm. Pure. Appl. Math. 35 (1982), 771-831.

[3] C. Cao and J. Wu, Two regularity criteria for the 3D MHD equations. J. Differential Equations 248 (2010), 2263-2274. 
[4] D. Chae, K. Kang and J. Lee, On the interior regularity of suitable weak solutions to the Navier-Stokes equations. Comm. Partial Differential Equations 32 (2007), 1189-1207.

[5] J. Chemin, B. Desjardin, I. Gallagher and E. Grenier, Fluids with anisotropic viscosity. Special issue for R. Temam's 60th birthday. M2AN Math. Model. Numer. Anal. 34 (2000), 315-335.

[6] H. Dong and X. Gu, Partial Regularity of solutions to the Four-Dimensional Navier-Stokes Equations. To appear in Dynamics of PDE. See also arXiv:1302.1443.

[7] S. Gustafson, K. Kang and T. Tsai, Interior regularity criteria for suitable weak solutions of the Navier-Stokes equations, Commun. Math. Phys. 273 (2007), 161-176.

[8] O. Ladyzenskaja and G. Seregin, On partial regularity of suitable weak solutions to the threedimensional Navier-Stokes equations, J. Math. Fluid Mech. 1 (1999), 356-387.

[9] F. Lin, A new proof of the Caffarelli-Kohn-Nirenberg Theorem, Comm. Pure Appl. Math. 51 (1998), 241-257.

[10] A. Majda and A. Bertozzi, Vorticity and incompressible flow. Cambridge Texts in Applied Mathematics, 27. Cambridge University Press, Cambridge, 2002.

[11] C. Miao and X. Zheng, On the global well-posedness for the Boussinesq system with horizontal dissipation. Comm. Math. Phys. 321 (2013), 33-67.

[12] J. Neustupa and P. Penel, Regularity of a suitable weak solution to the Navier-Stokes equations as a consequence of a regularity of one velocity component. In: H. Beirao da Veiga, A. Sequeira, J. Videman, Nonlinear Applied Analysis. New York: Plenum Press, (1999), 391-402.

[13] L. Nirenberg, An extended interpolation inequality. Ann. Scuola Norm. Sup. Pisa 20 (1966), 733-737.

[14] V. Scheffer, Partial regularity of solutions to the Navier-Stokes equations, Pacific J. Math. 66 (1976), 535-552.

[15] V. Scheffer, Hausdorff measure and the Navier-Stokes equations, Comm. Math. Phys. 55 (1977), 97-112.

[16] V. Scheffer, The Navier-Stokes equations on a bounded domain, Commun. Math. Phys. 73 (1980), 1-42.

[17] G. Seregin and V. Šverák, On smoothness of suitable weak solutions to the Navier-Stokes equations. (English, Russian summary) Zap. Nauchn. Sem. S.-Peterburg. Otdel. Mat. Inst. Steklov. (POMI) 306 (2003), Kraev. Zadachi Mat. Fiz. i Smezh. Vopr. Teor. Funktsii. 34, 186-198, 231; translation in J. Math. Sci. (N. Y.) 130 (2005), 4884-4892.

[18] G. Seregin, Local regularity theory of the Navier-Stokes equations. Handbook of mathematical fluid dynamics. Volume 4, Edited by S. Friedlander and D. Serre. North-Holland, Amsterdam, 2007, 159-200.

[19] G. Seregin, Estimates of suitable weak solutions to the Navier-Stokes equations in critical Morrey spaces. Zap. Nauchn. Sem. S.-Peterburg. Otdel. Mat. Inst. Steklov. (POMI) 336 (2006), Kraev. Zadachi Mat. Fiz. i Smezh. Vopr. Teor. Funkts. 37, 199-210, 277; translation in J. Math. Sci. (N. Y.) 143 (2007), 2961-2968.

[20] G. Seregin, On the local regularity of suitable weak solutions of the Navier-Stokes equations. (Russian) Uspekhi Mat. Nauk 62 (2007), 149- 168; translation in Russian Math. Surveys 62 (2007), 595-614.

[21] J. Serrin, On the interior regularity of weak solutions of the Navier-Stokes equations, Arch. Rational Mech. Anal. 9 (1962), 187-195. 
[22] R. Témam, Navier-Stokes equations: Theory and numerical analysis. AMS Chelsea Publishing, Providence, RI, 2001. Reprint of the 1984 edition.

[23] G. Tian and Z. Xin, Gradient estimation on Navier-Stokes equations, Comm. Anal. Geom. 7 (1999), 221-257.

[24] A. Vasseur, A new proof of partial regularity of solutions to Navier-Stokes equations, Nonlinear differ. equ. appl. 14 (2007), 753-785.

[25] W. Wang, L. Zhang and Z. Zhang, On the interior regularity criteria of the 3-D Navier-Stokes equations involving two velocity components. arXiv:1410.2399

[26] W. Wang and Z. Zhang, On the interior regularity criteria for suitable weak solutions of the magnetohydrodynamics equations. SIAM J. Math. Anal. 45 (2013), 2666-2677

[27] W. Wang and Z. Zhang, On the interior regularity criteria and the number of singular points to the Navier-Stokes equations, J. Anal. Math. 123 (2014), 139-170.

[28] Y. Wang and G. Wu, A unified proof on the partial regularity for suitable weak solutions of non-stationary and stationary Navier-Stokes equations. J. Differential Equations, 256 (2014), 1224-1249.

[29] X. Zheng, A regularity criterion for the tridimensional Navier-Stokes equations in term of one velocity component. J. Differential Equations 256 (2014), 283-309. 